\title{
A Round Robin Experiment: Analysis of Solute Clustering from Atom Probe Tomography Data.
}

Emmanuelle A. Marquis ${ }^{1}$, Vicente Araullo-Peters ${ }^{1}$, Aurianne Etienne ${ }^{2}$, Svetlana Fedotova ${ }^{3}$, Katsuhiko Fujii $^{4}$, Koji Fukuya ${ }^{4}$, Evgenia Kuleshova ${ }^{3}$, Anabelle Legrand ${ }^{5}$, Andrew London ${ }^{6}$, Sergio Lozano-Perez ${ }^{6}$, Yasuyoshi Nagai ${ }^{7}$, Kenji Nishida ${ }^{8}$, Bertrand Radiguet ${ }^{2}$, Daniel Schreiber ${ }^{9}$, Naoki Soneda ${ }^{8}$, Mattias Thuvander ${ }^{10}$, Takeshi Toyama ${ }^{6}$, Faiza Sefta ${ }^{11}$ and Peter Chou ${ }^{12}$

1. Department of Materials Science and Engineering, University of Michigan, Ann Arbor, MI, U.S.A.

2. Groupe de Physique des Matériaux, UMR CNRS 6634, Université de Rouen, Saint Etienne du Rouvray Cedex, France

4. NRC "Kurchatov Institute", Moscow, Russia

3. Institute of Nuclear Safety System, Inc., Kyoto, Japan

5. Commissariat à l'Energie Atomique (CEA), Saclay, France

6. Department of Materials, University of Oxford, U.K.

7. Institute for Materials Research, Tohoku University, Oarai Japan

8. Materials Science Research Laboratory, Central Research Institute of Electric Power Industry, Nagasaka, Japan

9. Energy and Environment Directorate, Pacific Northwest National Laboratory, Richland, WA, U.S.A.

10. Department of Physics, Chalmers University of Technology, Chalmers, Sweden

11. Departement Métallurgie, EDF, Moret sur Loing, France

12. Electric Power Research Institute, Palo Alto, CA, U.S.A.

Because atom probe tomography (APT) provides three-dimensional reconstructions of small volumes by resolving atomic chemical identities and positions, it is uniquely suited to analyze solute clustering phenomena in materials. To this end, a number of approaches have been developed to extract clustering information from the 3D reconstructed dataset, e.g. [1-3] and numerous reports can be found applying these methods. However, results from clustering analyses can differ significantly from one report to another, questioning the reliability of APT to quantitatively describe solute clustering. In addition, details of the analyses are often not provided, preventing independent confirmation of the results. With the number of APT research groups growing quickly, it is necessary to be educated about and to have a common understanding of APT artifacts, current analysis tools, and methods for data reporting, in order to provide appropriate context to discussions of prior data, data analysis protocols, possible sources of errors, and variability.

To address these issues, a round robin was organized among ten different international institutions. The goal is to provide a consistent framework for the analysis of irradiated stainless steels using atom probe tomography. Through the development of more reliable and reproducible data analysis and communication, this project also aims to advance the understanding between irradiated microstructure and materials performance by providing more complete quantitative microstructural input for modeling. The results, methods, and findings of this round robin may apply to other clustering phenomena studied using APT, beyond the theme of radiation damage.

Two types of APT datasets were examined: (1) simulated datasets generated to contain a known size distribution of clusters of varying compositions and (2) experimental datasets obtained from neutron or proton-irradiated stainless steels. The simulated datasets were specifically designed to test the resolution 
of current analysis tools, to push the development of more robust analysis methods, to assess variability among the results, and to enable new data reporting schemes. The analysis methods and results will be discussed.

\section{References:}

[1] D Vaumousse, A Cerezo, PJ Warren. Ultramicroscopy 95 (2003) p.215.

[2] LT Stephenson, MP Moody, PV Liddicoat, SP Ringer. Microscopy and Microanalysis 13 (2007) p. 448.

[3] T Philippe, F De Geuser, S Duguay, W Lefebvre, O Cojocaru-Miredin, G Da Costa, D Blavette. Ultramicroscopy 109 (2009) p. 1304.

[4] The authors acknowledge partial financial support from EPRI and EDF-MAI.

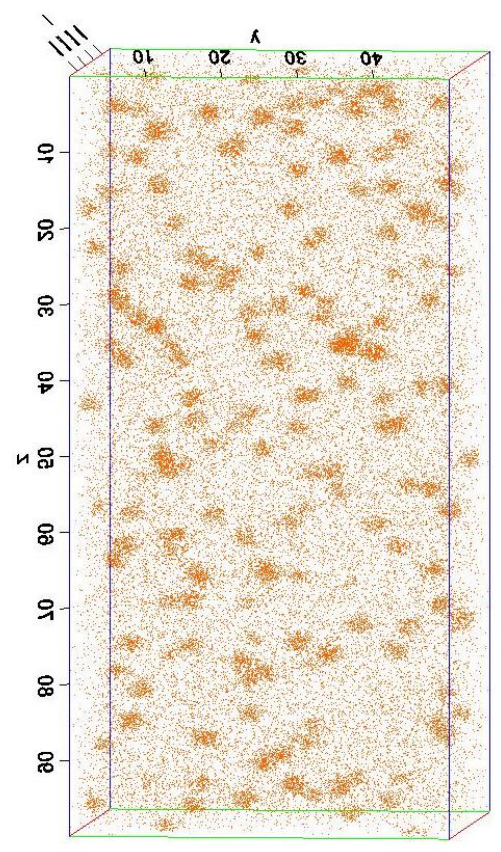

Figure 1: Simulated APT dataset containing 157 clusters of atom B with a target concentration of 70 at.\% B and a Gaussian size distribution. The overall dataset contains 0.695 at.\% B. 